\title{
Fatigue in COPD: association with functional status and hospitalisations
}

\author{
Johanna Susan Paddison*, Tanya W. Effing*,", Stephen Quinn* and Peter A. Frith*,\#
}

ABSTRACT: This study aimed to examine the importance of fatigue as a clinical indicator in chronic obstructive pulmonary disease (COPD), by analysing its relationship with COPD severity and ability to predict risk of hospitalisation, and by comparing the intensity of fatigue in stable COPD patients with levels of fatigue reported by patients with other chronic conditions.

We studied 100 consecutive patients attending assessment clinics before pulmonary rehabilitation. Both questionnaire and physiological data were collected. Partial correlations, multiple linear regressions and Cox proportional hazard models/negative binomial regressions were used to address the research questions.

A significant relationship existed between fatigue and COPD severity. Fatigue reports predicted future hospitalisation risk. Compared to the lowest third of patients, the third of patients reporting the most intense fatigue showed a 10-fold increase in risk of hospitalisation (fatigue experiences hazard ratio (HR) 10.2, 95\% $\mathrm{Cl}$ 2.66-38.86; fatigue impacts HR 10.7, 95\% $\mathrm{Cl} 2.76-41.65$ ). Our COPD sample reported fatigue scores of similar intensity to colorectal cancer patients and HIV-positive patients.

While fatigue is significantly related to COPD functional severity, fatigue data also capture independent information. Fatigue reports can contribute to predictions of hospitalisation risk.

KEYWORDS: BODE score, chronic obstructive pulmonary disease, fatigue

I $\mathrm{n}$ clinical interactions, fatigue frequently emerges as a prominent issue for patients living with chronic obstructive pulmonary disease (COPD). Fatigue has been reported as the second most prevalent symptom after dyspnoea [1]. Yet, the clinical significance of fatigue reports has received much less intensive investigation than other respiratory and systemic expressions of COPD, such as dyspnoea, decreases in body mass, exercise capacity and muscle strength, or increases in airflow obstruction. We therefore designed this study with three aims: to examine the relationship between fatigue and COPD severity; to investigate the ability of fatigue to predict risk of future hospitalisation; and to compare the intensity of fatigue in stable COPD patients with the levels of fatigue reported by patients with other chronic conditions.

Fatigue can refer to a physical or a mental state, or a combination of these. In this study, we assessed fatigue using a validated comprehensive questionnaire. The Identity-Consequences Fatigue Scale (ICFS), a self-report tool, assesses five domains of fatigue (feelings of fatigue, feelings of vigour, impacts on concentration, impacts on energy and impacts on daily activities) and provides two summary scores: fatigue experiences and fatigue impacts, [2, 3]. Because the ICFS is a generic fatigue questionnaire, it allows comparisons to be made between different patient populations.

Associations between fatigue and disease severity have been reported in other chronic diseases, such as chronic heart failure [4] and psoriatic arthritis [5]. Fatigue has been related to quality of life and functional limitation [6], but the association between fatigue and disease severity is less clear. Of the studies identified, several used the composite BODE score as a proxy for COPD severity $[7,8]$. The BODE score incorporates four components previously identified as independent predictors of prognosis in COPD: body mass, level of airflow obstruction, disablement due to dyspnoea and exercise capacity [9]. Its composite is a better prognostic index of outcome than any of its components.

The clinical value of fatigue in patients with COPD still needs to be determined. Might there be added value for the clinician to be more analytical when a patient reports fatigue? Previously, fatigue
AFFILIATIONS

*Dept of Respiratory Medicine, Repatriation General Hospital, and ${ }^{\#}$ School of Medicine, Flinders University, Adelaide, Australia.

CORRESPONDENCE

J.S. Paddison

Respiratory Function Unit Repatriation General Hospital Daws Road Daw Park Adelaide 5041 Australia E-mail: johanna.paddison@ health.sa.gov.au

Received: Feb 032012 Accepted after revision: May 212012 First published online: June 142012 
reports have been shown to predict the time to death of terminally ill lung cancer patients [10], but no data have been published about the predictive value of fatigue in patients with COPD.

In examining the importance of fatigue reports in COPD patients, we first examined whether patient-reported fatigue can predict the risk of future hospitalisation. Secondly, we examined the relationship between fatigue and severity of COPD. Finally, we compared the severity of fatigue measured using the ICFS in COPD patients to other chronic disease populations (patients with colorectal cancer and HIV).

\section{METHODS}

In this project, a sample of 100 consecutive patients were recruited. These patients were attending an assessment clinic prior to entry to a pulmonary rehabilitation programme at the Repatriation General Hospital, Adelaide, Australia (JanuaryDecember 2008). Ethical permission was gained from the Flinders Clinical Research Ethics Committee, Adelaide. Data collected included demographic variables, fatigue data, measures of COPD severity and prospectively recorded hospital admissions.

\section{Fatigue assessment}

The ICFS, a 31-item self-report tool, assesses five domains of fatigue (feelings of fatigue, feelings of vigour, impacts on concentration, impacts on energy and impacts on daily activities) and provides two summary scores: fatigue experiences and fatigue impacts, [2, 3]. The fatigue experiences score is the average of the feelings of fatigue, feelings of vigour and impacts on concentration subscales. The fatigue impacts score is the average of the impacts on energy and impacts on daily activities subscales. For this work, scores were generated on a scale from 0 to 10 (where a higher score indicates greater fatigue).

\section{Measures of COPD clinical state}

Level of airflow obstruction was obtained from measurement of the forced expiratory volume in $1 \mathrm{~s}$ (FEV1) using postbronchodilator efforts, according to American Thoracic Society (ATS)/European Respiratory Society quality performance criteria [11], and using prediction equations for sex, age and height [12]. Disablement due to breathlessness was assessed using the modified Medical Research Council (mMRC) dyspnoea scale [13]. This measure assesses dyspnoea on a five-point scale with descriptive anchors ranging from 0 ("not troubled by breathlessness except on strenuous exercise") to 4 ("too breathless to leave the house, or breathless when dressing or undressing"). Exercise capacity was obtained from the 6-min walk distance (6MWD), assessed using the ATS protocol; scoring was taken from metres walked [14]. Body mass index (BMI) was calculated as weight divided by squared height $\left(\mathrm{kg} \cdot \mathrm{m}^{-2}\right)$, obtained from fully clothed participants with shoes removed.

BODE scores were calculated according to the instructions of CELLI et al. [9]. Participants' BMIs were categorised as 0 $\left(\geqslant 21.1 \mathrm{~kg} \cdot \mathrm{m}^{-2}\right)$ or $1\left(\leqslant 21 \mathrm{~kg} \cdot \mathrm{m}^{-2}\right)$; their $\mathrm{FEV} 1 \%$ predicted values were scored as: $0: \geqslant 65 ; 1: 64-50 ; 2: 49-36$; and $3: \leqslant 35$; raw scores for mMRC were used (scores 0-4), as detailed above. Participants' 6MWDs were categorised as: 0: $\geqslant 350 \mathrm{~m} ; 1$ : 349-250 m; 2: 249-150 m; and 3: $\leqslant 149 \mathrm{~m}$. Following these ratings, the component scores were summed to give a BODE index score, covering the range from 0 (normal functional status) to 10 (maximal functional impairment).

\section{Comparison of COPD fatigue levels with two other chronic disease populations}

The ICFS data from respiratory patients with complete datasets $(n=83)$ were compared with two previously published datasets: 1) 38 colorectal cancer patients awaiting surgery recruited from outpatient clinics at Middlemore Hospital, Auckland, New Zealand [15]; and 2) 38 HIV-positive patients recruited from outpatient clinics at Massachusetts General Hospital, Boston, MA, USA [16].

\section{Hospital admission data}

Electronic hospital records at the patients' local tertiary centres (Flinders Medical Centre and Repatriation General Hospital) were examined. The period was 20 months from the date of study recruitment. Any in-patient admission with an International Statistical Classification of Diseases-10 code from the respiratory " $\mathrm{J}$ " group listed as the primary diagnosis was recorded [17]. The number of admissions and associated lengths of stay were recorded.

\section{Statistical analyses}

Partial correlations, adjusting for age and sex, were used to assess the associations between BODE and fatigue experiences and fatigue impacts. For this analysis fatigue impact scores were transformed by taking the square root to meet normality assumptions. Fatigue experiences and BODE were normally distributed.

Time to hospitalisation was investigated using Cox proportional hazard shared frailty models, clustering over individuals to account for correlated observations, and adjusting for age, sex, BODE and either fatigue experiences or fatigue impacts, i.e. the effects of fatigue experiences and fatigue impacts were examined in separate models. Initially, each model consisted of the fatigue type, BODE, age and sex, where fatigue type and BODE were entered as continuous variables, but the proportional hazard assumption was not met. Moreover, no transformation of the predictors of interest improved the model sufficiently. Therefore, fatigue experiences, fatigue impacts and BODE were trichotomised. The resulting models satisfied the proportional hazards assumption. Sensitivity analyses were also conducted and reported to examine the robustness of the results, where fatigue experiences, fatigue impacts and BODE were dichotomised and divided into quartiles. The associations between length of stay and each fatigue outcome were similarly assessed using multivariable negative binomial regression, adjusting for age, sex and BODE, to account for possible overdispersion.

To assess how the severity of fatigue in COPD related to other groups with chronic conditions, the mean levels of fatigue between groups were evaluated using multiple linear regression, adjusting for age and sex. Fatigue experiences and fatigue impacts were examined separately. Residual diagnostic checks for normality and homoscedasticity were conducted to ensure model validity. A p-value $<0.05$ (two-tailed) was considered statistically significant. All statistical analyses were performed on Intercooled Stata 11 for Windows (StataCorp, College Station, TX, USA). 


\section{RESULTS}

\section{Patient characteristics}

Of the 100 consecutive patients attending assessment clinics prior to entry to a pulmonary rehabilitation programme, missing data in the dyspnoea variable (mMRC score) precluded BODE score calculation in 17 of the patients examined; thus, only 83 complete respiratory patient datasets could be generated. These 83 patients constituted the sample analysed. No statistically significant differences were identified between those with complete data and those with missing mMRC scores. During the 20-month follow-up, six patients died. The mean \pm SD FEV1 of $53 \pm 21 \%$ pred shows that by Global Initiative for Chronic Obstructive Lung Disease criteria [18], the COPD patients, on average, had moderate disease, although there was a wide range of FEV1 severity (table 1). On average, our patient sample reported their dyspnoea to be between "walk slower than contemporaries on level ground because of breathlessness" and "have to stop for breath when

\section{TABLE 1 Patient characteristics}

\begin{tabular}{lc} 
COPD patients & 83 \\
Male & 49 \\
Age yrs & $72 \pm 9.3$ \\
Fatigue experiences & $4.7 \pm 1.6$ \\
Fatigue impacts & $4.0 \pm 1.9$ \\
FEV $\%$ pred & $51 \pm 21$ \\
GOLD class I & 24 \\
GOLD class II & 10 \\
GOLD class III & 31 \\
GOLD class IV & 18 \\
mMRC & $2.2 \pm 1.2$ \\
6MWD m & $388 \pm 115$ \\
BMI kg·m ${ }^{-2}$ & $27 \pm 5.3$ \\
BODE score & $3.5 \pm 2.4$ \\
HIV-positive patients & 38 \\
Male & 29 \\
Age yrs & $45 \pm 5.9$ \\
Fatigue experiences & $5.3 \pm 1.9$ \\
Fatigue impacts & $4.1 \pm 2.3$ \\
CD4 count cells·mm ${ }^{-3}$ & $526 \pm 33$ \\
Colorectal cancer patients & 38 \\
Male & 13 \\
Age yrs & $68 \pm 13.2$ \\
Fatigue experiences & $4.4 \pm 2.0$ \\
Fatigue impacts & $3.2 \pm 2.1$ \\
Duke's classification of disease extent & \\
A & \\
B & 4 \\
C & 9 \\
Unknown & 13 \\
& 12 \\
\hline
\end{tabular}

Data are presented as $n$ or mean $\pm S D$. COPD: chronic obstructive pulmonary disease; FEV 1 : forced expiratory volume in $1 \mathrm{~s}$; \% pred: \% predicted; GOLD: Global Initiative for Chronic Obstructive Lung Disease; mMRC: modified Medical Research Council dyspnoea scale; 6MWD: 6-min walk distance; BMI: body mass index; BODE: body mass, level of airflow obstruction, disablement due to dyspnoea, exercise capacity. ${ }^{\#}$ : it is known that no patient in the series had a diagnosis of metastatic cancer. walking at own pace" (mean mMRC 2.3 \pm 1.2 ). During the $6 \mathrm{MWD}$ test, $36 \%$ of the patients walked $<350 \mathrm{~m}(388 \pm 117 \mathrm{~m})$, with distances $<350 \mathrm{~m}$ considered to indicate levels of functional impairment of prognostic significance to mortality [19]. The mean BODE score was $3.5 \pm 2.4$. For the COPD patients, the mean fatigue experiences score was $48 \pm 17 \%$ of the available range; the mean fatigue impacts score was $41 \pm 21 \%$ of the available range.

$24 \%$ of the COPD patients had at least one hospital admission during the 20 months of follow-up; $58 \%$ of those hospitalised during follow-up were admitted on multiple occasions (range $1-11 \%)$. There were a total of 58 admissions. The median length of stay was 7 days (range 1-52 days, interquartile range 4-14 days).

For the COPD patients, significant relationships were present between fatigue experiences score and BODE score $\left(\mathrm{r}^{2}=0.38\right.$, $\mathrm{p}=0.001)$ and between fatigue impacts score and BODE score $\left(r^{2}=0.49, p=0.001\right)$. These associations are medium by the criteria of COHEN [20].

Multivariable analyses showed that for fatigue experiences, the most severely fatigued third of the sample was at greater risk of hospitalisation than the lowest (hazard ratio (HR) 11.4, 95\% CI 2.56-50.53; table 2) and middle thirds (HR 5.0 95\% CI 1.4018.12). Compared with the lowest third of patients, the third of patients reporting the most intense scores on fatigue impacts showed a 13.6-fold increase in risk of hospitalisation $(95 \% \mathrm{CI}$ 2.50-74.20). For fatigue impacts, no significant increase in risk of hospitalisation existed when the middle third of patients was compared to the two extremes. Sensitivity analyses showed that when the fatigue data were dichotomised, the more fatigued participants experienced higher rates of hospitalisation (fatigue experiences HR 5.4, 95\% CI 1.51-19.03; fatigue impacts HR 5.0, 95\% CI 1.24-20.44). The only significant differences identified in the quartiled data were between the lowest and third quartiles (fatigue experiences HR 10.2, 95\% CI 1.70-61.66; fatigue impacts HR 7.2, 95\% CI 1.17-44.78). Length of hospital stay increased by a factor of almost 4 for every unit increase in fatigue experiences (incidence rate ratio (IRR) 3.72, 95\% CI 1.374.84; table 3) and by over 2 for every unit increase in fatigue impacts (IRR 2.36, 95\% CI 0.43-2.90).

After controlling for age and sex differences, there was no significant difference between the ICFS fatigue scores of our population of COPD patients (fatigue experiences $4.7,95 \% \mathrm{CI}$ 3.7-5.8; fatigue impacts $4.2,95 \%$ CI 3.0-5.4) and the populations of patients with colorectal cancer awaiting surgery (fatigue experiences $4.5,95 \%$ CI 3.5-5.5; fatigue impacts 3.5, 95\% CI 2.3-4.7) and HIV-positive patients (fatigue experiences 5.3, 95\% CI 3.9-6.7; fatigue impacts 4.5 , 95\% CI 2.9-6.1).

\section{DISCUSSION}

From the patient's perspective, fatigue is a prominent element in the symptoms experienced as a consequence of COPD [1]. However, there is little quantitative evidence supporting the clinical relevance of fatigue reports. Beyond clinical "gut feeling", clinicians have few sources of information to guide their interpretation of what patients mean when they report subjective fatigue. This study confirms that fatigue reports are systematically related to markers of COPD disease severity. Of greater clinical importance, our results show that, independent 
TABLE 2 Risk of hospitalisation in chronic obstructive pulmonary disease patients over 20 months

\begin{tabular}{lcc} 
& Multivariate models \\
\hline Fatigue experiences model & Univariate analyses & \\
Fatigue experiences highest versus lowest third & $18.24(4.00-83.67)^{\star * *}$ & $11.37(2.56-50.53)^{* * *}$ \\
Fatigue experiences middle versus lowest third & $3.29(0.88-12.30)$ & $2.25(0.58-8.71)$ \\
BODE highest versus lowest third & $5.83(0.20-167.43)$ & $5.87(1.25-27.60)^{*}$ \\
BODE middle versus lowest third & $3.04(0.76-12.21)$ & $3.05(0.70-13.25)$ \\
Age & $0.75(0.27-2.05)$ & $0.98(0.93-1.04)$ \\
Male & $1.88(0.53-6.59)$ & $1.17(0.37-3.70)$ \\
Fatigue impacts model & & $13.63(2.50-74.20)^{\star *}$ \\
Fatigue impacts highest versus lowest third & $22.25(4.38-113.48)^{\star * *}$ & $4.30(0.77-24.01)$ \\
Fatigue impacts middle versus lowest third & $4.25(0.90-20.08)$ & $2.70(0.49-14.82)$ \\
BODE highest versus lowest third & $5.83(0.20-167.43)$ & $2.18(0.45-10.58)$ \\
BODE middle versus lowest third & $3.04(0.76-12.21)$ & $0.99(0.92-1.06)$ \\
Age & $0.75(0.27-2.05)$ & $1.98(0.54-7.23)$ \\
Male & $1.88(0.53-6.59)$ & \\
\hline
\end{tabular}

Data are presented as hazard ratio $(95 \% \mathrm{Cl}) . \mathrm{n}=83$. BODE: body mass, level of airflow obstruction, disablement due to dyspnoea and exercise capacity. ${ }^{*}$ : $<<0.05$; ${ }^{* *}: p<0.01 ;{ }^{* *}: p<0.001$.

of disease severity, fatigue is a strong predictor of hospitalisation risk, which is itself an important driver of impaired quality of life, negative impact on self and family, escalation of personal and societal costs, and ongoing instability and mortality.

It is well established that COPD patients experience high rates of hospital admission [21, 22], inducing a substantial economic burden [23, 24]. Identifying signals that predict future hospitalisation risk in COPD patients is therefore of both clinical and economic relevance. There is mounting evidence that BODE scores predict outcomes such as hospitalisations and mortality [25-27]. Our work shows that fatigue data independently predicts hospitalisation risk.

\section{TABLE 3 \\ Predicting length of hospital admission in chronic obstructive pulmonary disease patients}

\begin{tabular}{lcc} 
& Univariate analyses & Multivariate models \\
\hline Fatigue experiences & & \\
model & & \\
Fatigue experiences & $4.19(2.75-6.36)^{\star \star \star}$ & $3.72(2.37-5.84)^{\star \star *}$ \\
BODE & $1.56(0.77-3.17)$ & $1.42(0.81-2.47)$ \\
Age & $0.50(0.31-0.82)^{*}$ & $0.85(0.44-1.65)$ \\
Male & $1.53(0.33-7.0)$ & $2.11(0.81-5.49)$ \\
Fatigue impacts & & \\
model & & \\
Fatigue impacts & $2.48(1.46-4.21)^{\star \star *}$ & $2.36(1.43-3.90)^{\star * *}$ \\
BODE & $1.56(0.77-3.17)$ & $1.21(0.51-2.90)$ \\
Age & $0.50(0.31-0.82)^{\star}$ & $0.88(0.42-1.84)$ \\
Male & $1.53(0.33-7.0)$ & $4.29(1.12-16.50)^{*}$ \\
\hline
\end{tabular}

Data are presented as incidence rate ratio $(95 \% \mathrm{Cl}) . \mathrm{n}=83$. BODE: body mass, level of airflow obstruction, disablement due to dyspnoea and exercise capacity. ${ }^{*}: p<0.05 ;{ }^{* * *}: p<0.001$.
In the model including fatigue experiences, a BODE score within the upper extreme third was associated with a hospitalisation risk five times greater than the 20-month risk for those with a BODE score within the lowest third. Comparisons between the extreme thirds of the fatigue data identified 11- and 13-fold differences in the risk of hospitalisation for fatigue experiences and fatigue impacts respectively. Post hoc analyses examining the relationship between the fatigue data and length of hospital stay also supported the strong discriminating role of fatigue reports regarding duration of hospitalisations.

We found significant relationships between fatigue and disease severity, as measured by BODE, in COPD patients attending pulmonary rehabilitation. As expected, when BODE scores increased so did fatigue scores. Causal mechanisms explaining this are likely, at least in part, to lie in the growing evidence suggesting that COPD causes a state of systemic inflammation $[28,29]$. It is well recognised that inflammation can induce a behavioural profile termed "sickness behaviour" [30]. In humans, cytokine-induced sickness behaviour includes subjective reports of lethargy and tiredness [31], while experimental animal models show behavioural markers of fatigue [32]. While some people with COPD have measurable systemic inflammation out of proportion to the severity of their airflow limitation, there is an overall association between COPD severity and derangement of inflammatory processes [33, 34]. There are, of course, other explanations for fatigue in COPD patients, such as the high oxygen cost of breathing, especially in hyperinflated patients, or the limiting effect of hypoxaemia on activities.

Existing literature has shown that COPD patients report greater fatigue than the general population [35-37]. However, the severity of fatigue between COPD patients and other clinical populations has rarely been examined. Fatigue experiences and impacts reported by our COPD population were of similar intensity to those reported by colorectal cancer patients 
awaiting surgery and by HIV-positive patients [11, 12]. The occurrence of chronic inflammation, which is common to these three situations [38, 39], may help to explain this observation. Other common explanations may include fear and other psychological reactions to potentially or ultimately fatal diseases, or factors such as sleep disturbance and disruption to circadian rhythms $[40,41]$.

There are several limitations to the current study. The COPD patients are all patients who were included in a pulmonary rehabilitation programme and, therefore, a selection of more symptomatic COPD patients. The results of this study may not apply to the whole COPD population. Also, admissions to private hospitals or to hospitals other than the patients' local tertiary centres were not recorded. However, given the geography of Adelaide and general patient flows, we anticipate few cases would have been affected by this issue. Finally, it is known that pulmonary rehabilitation influences admission rates in patients with COPD $[42,43]$, so it is possible that our admission rates will be lower than a population that has not attended pulmonary rehabilitation.

Overall, more research is needed to understand the clinical meaning attributable to patient reports of fatigue. Our results, particularly those relating to hospitalisation risk, while suggesting clinical importance, require replication. Work to investigate potential causal mechanisms is also of importance. Other directions for future research include establishment of ICFS norm values using a healthy sample in adults and elderly patients, and investigation of whether the ICFS tool is sensitive enough to capture changes in fatigue reports during periods of clinical change, such as during infective exacerbations of COPD or following interventions such as pharmacological treatments or pulmonary rehabilitation. Understanding the relationship between fatigue and BODE will be of special importance. Our data show that fatigue and BODE scores share only $30-50 \%$ of their variation, which implies that fatigue reports capture information about patient state that is not summarised in the BODE index and vice versa. Identifying what underlies this unshared element of variance will be important to understanding how fatigue contributes to hospitalisation risk separately from BODE.

In conclusion, this study has shown that fatigue reports are significantly related to COPD severity in COPD patients attending a pulmonary rehabilitation programme and also that fatigue scores capture additional information. Our sample of COPD patients reported fatigue intensity that was similar to that reported by colorectal cancer patients and by HIV-positive patients. Finally, fatigue reports were shown to significantly predict hospitalisation risk and length of hospital admission.

\section{STATEMENT OF INTEREST}

A statement of interest for P.A. Frith can be found at www.erj. ersjournals.com/site/misc/statements.xhtml

\section{REFERENCES}

1 Blinderman CD, Homel P, Billings JA, et al. Symptom distress and quality of life in patients with advanced chronic obstructive pulmonary disease. J Pain Symptom Manage 2009; 38: 115-123.
2 Paddison JS, Hill AG, Cameron LD, et al. Comprehensive assessment of post-surgical fatigue: development of the IdentityConsequence Fatigue Scale. J Psychosom Res 2006; 60: 615-622.

3 Paddison JS. Post-surgical fatigue: psychological and physiological predictors of fatigue trajectory following major colorectal surgery. PhD thesis. The University of Auckland, Auckland, 2007.

4 Falk K, Swedberg K, Gaston-Johansson F, et al. Fatigue is a prevalent and severe symptom associated with uncertainty and sense of coherence in patients with chronic heart failure. Eur J Cardiovasc Nurs 2007; 6: 99-104.

5 Husted JA, Tom BD, Farewell VT, et al. Longitudinal analysis of fatigue in psoriatic arthritis. J Rheumatol 2010; 37: 1878-1884.

6 Theander K, Jakobsson P, Torstensson O, et al. Severity of fatigue is related to functional limitation and health in patients with chronic obstructive pulmonary disease. Int J Nurs Pract 2008; 14: 455-162.

7 Marin JM, Cote CG, Diaz O, et al. Prognostic assessment in COPD: health related quality of life and the BODE index. Respir Med 2011; 105: 916-921.

8 Al-shair K, Kolsum U, Berry P, et al. Development, dimensions, reliability and validity of the novel Manchester COPD fatigue scale. Thorax 2009; 64: 950-955.

9 Celli BR, Cote CG, Marin JM, et al. The body-mass index, airflow obstruction, dyspnea, and exercise capacity index in chronic obstructive pulmonary disease. N Engl J Med 2004; 350: 1005-1012.

10 Paddison JS, Temel JS, Fricchione GL, et al. Using the differential from complete blood counts as a biomarker of fatigue in advanced non-small-cell lung cancer: an exploratory analysis. Palliat Support Care 2009; 7: 213-217.

11 Miller MR, Hankinson J, Brusasco V, et al. Standardisation of spirometry. Eur Respir J 2005; 26: 319-338.

12 Qanjer PH, Tammeling GJ, Cotes JE, et al. Lung volumes and forced ventilatory flows. Report Working Party Standardization of Lung Function Tests, European Community for Steel and Coal. Official Statement of the European Respiratory Society. Eur Respir J 1993; 6: Suppl. 16, 5-40.

13 Mahler DA, Wells CK. Evaluation of clinical methods for rating dyspnea. Chest 1988; 93: 580-586.

14 Crapo RO, Casaburi R, Coates AL, et al. ATS statement: guidelines for the six-minute walk test. Am J Respir Crit Care Med 2002; 166: 111-117.

15 Paddison J, Booth RJ, Cameron LD, et al. Fatigue after colorectal surgery and its relationship to patient expectations. J Surg Res 2009; 151: 145-152.

16 Paddison J, Fricchione G, Gandhi R, et al. Fatigue in psychiatric HIV patients: a pilot study of psychological correlates. Psychosomatics 2009; 50: 455-460.

17 World Health Organisation. ICD-10 Classifications of Mental and Behavioural Disorder: Clinical Descriptions and Diagnostic Guidelines. Geneva, World Health Organization, 1992.

18 Rabe KF, Hurd S, Anzueto A, et al. Global strategy for the diagnosis, management, and prevention of chronic obstructive pulmonary disease: GOLD executive summary. Am J Respir Crit Care Med 2007; 176: 532-555.

19 Cote CG, Casanova C, Marin JM, et al. Validation and comparison of reference equations for the 6-min walk distance test. Eur Respir J 2008; 31: 571-578.

20 Cohen J. Statistical Power Analysis for the Behavioral Sciences. Hillsdale, Lawrence Erlbaum, 1988.

21 Mannino DM, Homa DM, Akinbami LJ, et al. Chronic obstructive pulmonary disease surveillance - United States, 1971-2000. MMWR Surveill Summ 2002; 51: 1-16.

22 Kinnunen T, Saynajakangas O, Keistinen T. The COPD-induced hospitalization burden from first admission to death. Respir Med 2007; 101: 294-299.

23 Access Economics, Australian Lung Foundation. Economic Impact of COPD and Cost-Effective Solutions. www.lungfoundation.com. $\mathrm{au} /$ images/stories/docs/copd/2008_alf_access_economic_impact_ report.pdf 
24 Mannino DM, Braman S. The epidemiology and economics of chronic obstructive pulmonary disease. Proc Am Thorac Soc 2007; 4: 502-506.

25 Ko FW, Tam W, Tung AH, et al. A longitudinal study of serial BODE indices in predicting mortality and readmissions for COPD. Respir Med 2011; 105: 266-273.

26 Marin JM, Carrizo SJ, Casanova C, et al. Prediction of risk of COPD exacerbations by the BODE index. Respir Med 2009; 103: 373-378.

27 Celli BR. Predictors of mortality in COPD. Respir Med 2010; 104: 773-779.

28 Gan WQ, Man SFP, Senthilselvan A, et al. Association between chronic obstructive pulmonary disease and systemic inflammation: a systematic review and a meta-analysis. Thorax 2004; 59: 574-580.

29 Nussbaumer-Ochsner Y, Rabe KF. Systemic manifestions of COPD. Chest 2011; 189: 165-178.

30 Konsman JP, Parnet P, Danzter R. Cytokine-induced sickness behaviour: mechanisms and implications. Trends Neurosci 2002; 25: 154-159.

31 Kelley KW, Bluthe R-M, Danzter R, et al. Cytokine-induced sickness behavior. Brain Behav Immun 2003; 17: S112-S118.

32 Aubert A. Sickness and behaviour in animals: a motivational perspective. Neurosci Biobehav Rev 1999; 23: 1029-1036.

33 Thorleifsson SJ, Margretardottir OB, Gudmundsson G, et al. Chronic airflow obstruction and markers of systemic inflammation: results from the BOLD study in Iceland. Respir Med 2009; 103: 1548-1553.

34 Donaldson GC, Seemungal TAR, Patel IS, et al. Airway and systemic inflammation and decline in lung function in patients with COPD. Chest 2005; 128: 1995-2000.
35 Lewko A, Bidgood PL, Garrod R. Evaluation of psychological and physiological predictors of fatigue in patients with COPD. Pulm Med 2009; 9: 47-58.

36 Baghai-Ravary R, Quint JK, Goldring JJ, et al. Determinants and impact of fatigue in patients with chronic obstructive pulmonary disease. Respir Med 2009; 103: 216-223.

37 Theander K, Jakobsson $\mathrm{P}$, Torstensson $\mathrm{O}$, et al. Severity of fatigue is related to functional limitation and health in patients with chronic obstructive pulmonary disease. Int J Nurs Pract 2008; 14: 455-462.

38 Kemik O, Sumer A, Kemik AS, et al. The relationship among acutephase response proteins, cytokines and hormones in cachectic patients with colon cancer. World J Surg Oncol 2010; 8: 85-91.

39 Nixon DE, Landay AL. Biomarkers of immune dysfunction in HIV. Curr Opin HIV AIDS 2010; 5: 498-503.

40 Roscoe JA, Kaufman ME, Matteson-Rusby SE, et al. Cancer-related fatigue and sleep disorders. Oncologist 2007; 12: 35-42.

41 Henderson M, Safa F, Easterbrook P, et al. Fatigue among HIVinfected patients in the era of highly active antiretroviral therapy. HIV Med 2005; 6: 347-352.

42 Raskin J, Spiegler P, McCusker C, et al. The effect of pulmonary rehabilitation on healthcare utilization in chronic obstructive pulmonary disease: the northeast pulmonary rehabilitation consortium. J Cardiopulm Rehabil 2006; 26: 231-236.

43 Hui KP, Hewitt AB. A simple pulmonary rehabilitation program improves health outcomes and reduces hospital utilization in patients with COPD. Chest 2003; 124: 94-97. 